\title{
Study on the Relationship Between Hypertension and Psycho-social Factors in Children and Adolescents
}

\author{
Chen Yun ${ }^{1}$
}

\author{
${ }^{1}$ Wannan Medical College, Wuhu city, Anhui Province \\ Author's profile: lecturer, Master of Psychology,785101592@qq.com
}

\begin{abstract}
Research Objective:This study aims at exploring the psycho-social factors inducing hypertension in children and adolescents, so as to provide reference for the intervention and treatment of hypertension in the young generation. Method: This article measures the blood pressure level, mental health status (GHQ-28) and life satisfaction of 2201 teenagers aged from 13 to 18 in middle schools in Wuhu. Logistic regression is utilized to analyze the psycho-social factors related to hypertension in teenagers. Findings: among 2201 surveyed respondents, 209 of them are diagnosed with hypertension, with an overall detection rate of $9.5 \%$. The total scores of mental health status, social dysfunction and severe depression are higher in teenagers with hypertension than those without, while the school satisfaction scores are lower than that of the group without hypertension. The disparity between the two groups has statistical significance $(p<0.05)$. As is showed in multivariate unconditional logistic regression analysis, rural areas upbringing and mental health status positive play a hazardous role in inducing hypertension in children and adolescents. Conclusion: Affected by psycho-social factors, hypertension in teenagers has a high detection rate. Therefore, psychological education and intervention should be carried out specifically for children and adolescents with hypertension, so as to alleviate negative emotions and pressure, improve social functions, mental health and effectively prevent and control the hypertension in teenagers.
\end{abstract}

Keywords: children and adolescents, hypertension, psychosocial factors

\section{FOREWORD}

As a well-known psychosomatic disorder, hypertension has a very high morbidity rate worldwide, with an upward trend at a younger age [1] The prevalence rate of hypertension in Chinese children and adolescents ranges from $0.5 \%$ to $20.3 \%$. As is showed in multiple researches, hypertension in adolescence can develop into that in adulthood as well, namely the blood pressure tracking phenomenon. Therefore, it is very necessary to conduct researches on hypertension in teenagers. This research aims to analyze the contributing factors of hypertension in children and adolescents from the perspective of social psychology, in the hope of providing a theoretical basis for the early prevention of hypertension and targeted correction of psychological behaviors.

\section{OBJECTS AND METHODS}

\subsection{Objects}

With convenient cluster sampling method, we have investigated 2332 students aged from 13 to 18 in middle schools in Wuhu City. After screening the research contents in this subject, 2201 valid questionnaires have been collected, including 1155 male respondents, 1046 females, 1534 urban people, 667 rural people, 1051 senior primary school students, 683 junior high school students and 467 senior high school students.

\subsection{Measurement work and assessment method}

Firstly, compile questionnaires, including gender, age, region, mother/father's education background, family annual income, etc.

Secondly, measure blood pressure level of respondents. Yuwell YE655A arm electronic sphygmomanometer has been uniformly used for measurement. The assessment method: hypertension is assessed when the systolic and/or diastolic pressure are consistently higher than or equal to the 95th percentile (P95) of the blood pressure for the same sex in the age group.

Thirdly, general health status (GHQ-28) scale. This scale is widely used in the medical evaluation of mental health status.

Fourthly, adolescent life satisfaction scale (CASLSS). This scale includes six dimensions: family satisfaction, friendship satisfaction, academic satisfaction, school satisfaction, freedom satisfaction and environment satisfaction. 


\subsection{Statistical method}

SPSS16.0 is used to process the data.The independent sample t-test method is used to compare the GHQ and life satisfaction of both hypertension group and non-hypertension group of teenagers, and $\chi^{2}$ test is used to compare hypertension relevance ratio.Meanwhile, univariate/multivariate unconditional logistic regression analysis is used to analyze the hypertension influencing factors, $\alpha=0.05$.

\section{FINDINGS}

\subsection{Basic findings of blood pressure measurement, GHQ and life satisfaction survey for children and adolescents}

Among the 2201 children and adolescents, 209 are diagnosed with hypertension in the blood pressure measurement, with overall detection rate of $9.5 \%$. There is a statistical significance in the detection rate comparison of hypertension among children and adolescents in different regions, grades, mothers' education background and fathers' education background $(p<0.05)$. Please see details in table 1 .

As is showed in GHQ-28 survey, there are 749 people with positive health status, positive incidence reaching $34.0 \%$.

The life satisfaction of adolescents generally stays in a basic satisfaction state (the average value of each question is 4.93). Among this, freedom satisfaction, academic satisfaction and environment satisfaction score very low. With the rise of grade, children and adolescents' life satisfaction gradually decreases. The life satisfaction of senior primary school students (185.02 \pm 34.18$)$, junior middle school students $(172.48 \pm 36.31)$, and senior high school students $(167.70 \pm 37.83)$ are statistically significant in difference by $\mathrm{F}$ test. $(\mathrm{F}=47.872, p<0.05)$.

Table 1 Comparison of Basic Information of Hypertension in Children and Adolescents

\begin{tabular}{|c|c|c|c|c|c|}
\hline & Total & Hypertension cases & Detection rate $(\%)$ & $\chi^{2}$ & $p$ \\
\hline \multicolumn{6}{|l|}{ gender } \\
\hline Male & 1155 & 104 & 9.0 & 0.683 & 0.409 \\
\hline Female & 1046 & 105 & 10.0 & & \\
\hline \multicolumn{6}{|l|}{ Residence } \\
\hline Urban & 1534 & 115 & 7.5 & 23.536 & 0.000 \\
\hline Rural & 667 & 94 & 14.1 & & \\
\hline \multicolumn{6}{|l|}{ Grades } \\
\hline senior primary & 1051 & 83 & 7.9 & 9.506 & 0.009 \\
\hline junior & 683 & 84 & 12.3 & & \\
\hline senior & 467 & 42 & 9.0 & & \\
\hline \multicolumn{6}{|l|}{ Mothers'diploma } \\
\hline illiteracy & 60 & 6 & 10.0 & 10.794 & 0.029 \\
\hline Primary school & 271 & 40 & 14.8 & & \\
\hline Junior school & 852 & 73 & 8.6 & & \\
\hline Senior/Secondary & 625 & 59 & 9.4 & & \\
\hline Junior college & 393 & 31 & 7.9 & & \\
\hline \multicolumn{6}{|l|}{ Fathers'diploma } \\
\hline illiteracy & 29 & 1 & 3.5 & 29.371 & 0.000 \\
\hline Primary school & 140 & 19 & 13.6 & & \\
\hline Junior school & 693 & 68 & 9.8 & & \\
\hline Senior/Secondary & 750 & 68 & 9.1 & & \\
\hline Junior college & 589 & 19 & 3.2 & & \\
\hline
\end{tabular}

\subsection{Comparison of $\mathrm{GHQ}$ and life satisfaction} between hypertensive and non-hypertensive teenagers dysfunction and severe depression in the hypertension group are higher than those in the non-hypertension group, while the score of academic satisfaction was lower than that in the non-hypertension group, with statistical significance $(\mathrm{P}<0.05)$.

Table 2 showed that the total score of GHQ,social

Table 2 Comparison of GHQ-28 and life satisfaction between hypertensive and non-hypertensive children and adolescents

\begin{tabular}{ccccc}
\hline Item & Hypertension & Non-hypertensive & $\mathrm{t}$ & $\mathrm{p}$ \\
\hline GHQscore & $4.88 \pm 4.98$ & $4.06 \pm 5.25$ & 2.25 & $.025^{*}$ \\
Somatization & $1.35 \pm 1.54$ & $1.17 \pm 1.58$ & 1.57 & .116 \\
Anxiety and Insomnia & $1.34 \pm 1.69$ & $1.21 \pm 1.80$ & 1.02 & .309 \\
Social dysfunction & $1.08 \pm 1.46$ & $0.83 \pm 1.45$ & 2.31 & $.021^{*}$ \\
severe depression & $1.11 \pm 1.73$ & $0.84 \pm 1.77$ & 2.05 & $.041^{*}$ \\
Score of Life Satisfaction & $175.26 \pm 34.79$ & $177.68 \pm 36.56$ & 0.36 & .361 \\
Family Satisfaction & $37.25 \pm 8.93$ & $37.24 \pm 9.05$ & 0.012 & .990 \\
Academic Satisfaction & $23.15 \pm 7.75$ & $24.33 \pm 7.97$ & 2.03 & $.043^{*}$ \\
Freedom Satisfaction & $22.82 \pm 6.73$ & $23.33 \pm 6.69$ & 1.04 & .298 \\
School Satisfaction & $31.00 \pm 7.93$ & $31.50 \pm 7.51$ & 0.93 & .350 \\
Environment Satisfaction & $25.57 \pm 5.65$ & $25.61 \pm 5.81$ & 0.09 & .929 \\
\hline
\end{tabular}




\subsection{Univariate Logistic Regression Analysis of Hypertension and Psycho-social Factors in Children and Adolescents}

According to high blood pressure $(\mathrm{y}=1, \mathrm{n}=0)$, gender $(m=1, f=2)$, region $(=1$ in cities, rural $=2)$, mother/father's degree $(=2$ illiteracy $=1$, elementary school, junior high school $=3$, high school or technical secondary school $=4$, college and above $=5$ ), annual family income $(<10000=1=2,3,1$ to $30000-60000=$
$3,>60000=4)$, relationship with their parents $(=1$, general $=2$, bad $=3$ ), mental health GHQ (negative $=1$, positive $=2$ ), life satisfaction and satisfaction in each field (basic not satisfied $=1$, partial satisfaction $=2$, the basic satisfaction $=3$, Very satisfied $=4$ ), assign a value to the study variable. Among the 13 variables selected, hypertension in children and adolescents was associated with regional, social dysfunction, severe depression, positive mental health status, school satisfaction, and mother's educational background. See Table 3 for details.

Table 3 Univariate Logistic Regression Analysis of Hypertension and Psycho-social Factors

\begin{tabular}{|c|c|c|c|c|c|}
\hline Variable & $\bar{\beta}$ & $\operatorname{SE}(\beta)$ & Wald & $\bar{P}$ & $\mathrm{OR}(95 \% \mathrm{Cl})$ \\
\hline Residence & 0.705 & 0.148 & 22.828 & 0.000 & $2.124(1.516 \sim 2.703)$ \\
\hline Social dysfunction score & 0.102 & 0.045 & 5.263 & 0.022 & $1.108(1.015 \sim 1.209)$ \\
\hline Severe depression score & 0.075 & 0.037 & 4.147 & 0.042 & $1.078(1.003 \sim 1.158)$ \\
\hline GHQ evaluation & 0.748 & 0.146 & 26.121 & 0.000 & $2.113(1.586 \sim 2.816)$ \\
\hline Academic satisfaction & -0.176 & 0.078 & 5.166 & 0.023 & $0.838(0.720 \sim 0.976)$ \\
\hline Mothers' diploma & -0.146 & 0.072 & 4.125 & 0.042 & $0.864(0.751 \sim 0.995)$ \\
\hline
\end{tabular}

\subsection{Multivariate Logistic regression analysis of hypertension and psycho-social factors in children and adolescents}

On the basis of univariate analysis, the variables multivariate Logistic regression analysis. Table 4 is a regression model fitted, and the regression model has statistical significance. Rural areas and positive mental health status are the risk factors of hypertension in children and adolescents.

Table 4 Multivariate Logistic regression analysis of hypertension and psychosocial related factors

\begin{tabular}{cccccc}
\hline Variable & $\beta$ & $\mathrm{SE}(\beta)$ & Wald & $\mathrm{P}$ & $\mathrm{OR}(95 \% \mathrm{Cl})$ \\
\hline Residence & 0.669 & 0.149 & 20.272 & 0.000 & $1.953(1.459 \sim 2.614)$ \\
GHQ evaluation & 0.716 & 0.147 & 23.634 & 0.000 & $2.046(1.533 \sim 2.713)$ \\
\hline
\end{tabular}

\section{DISCUSSIONS}

\subsection{Hypertension, mental health status and life satisfaction of children and adolescents}

Studies at home and abroad have shown that the morbidity of hypertension tends to be younger, and the prevalence of hypertension in children and adolescents is significantly higher, indicating an upward trend. This survey showed that the total detection rate of hypertension in children and adolescents was $9.5 \%$, among which the detection rate of boys was $9.0 \%$ and that of girls was $10.0 \%$. Compared with $12.4 \%$ of 35,657 children and adolescents aged 6 to 17 in 31 provinces (autonomous regions and municipalities directly under the central government) and 150 counties (districts) surveyed by Yu Dongmei et al, the prevalence of hypertension was lower, and $8.9 \%$ of 19,488 children and adolescents aged 7 to 17 was higher than that of Xi B's research [2]. The reasons for the inconsistency include the inconsistent assessment standards of hypertension in children and adolescents, the different cultural habits in different regions and the different levels of blood pressure prevention and control.

Relevant studies show that about $14.7 \%$ of the 340 million children and adolescents under the age of 17 in China suffered from various learning, emotional and behavioral disorders, and their mental health prospect is not optimistic. Zhang Hongbo et al. investigated 12,430 middle school students in Anhui Province and found that the incidence rate of psychological symptoms of depression was $22.8 \%$. A study by Yang Wenhui et al. of 2,731 adolescents in Yueyang City, Hunan Province showed that the prevalence of depressive symptoms was $22.81 \%$. In this study, GHQ-28 was used to evaluate the mental health status, and the overall positive rate of mental health status was $34.0 \%$, which was higher than the results in other literature, which may be caused by different sampling and measurement tools.

This study shows that children and adolescents are basically in a satisfied state of life satisfaction, but life 
satisfaction decreases with the increase of grade, which is similar to most previous studies.

\subsection{The influence of mental health, residence and other contributing factors in hypertension in children and adolescents}

It is widely recognized that there is a significant correlation between the occurrence of hypertension and mental health status. Most studies have pointed out the physiological mechanism of the adverse effect of depression on causing hypertension. This study also found that social dysfunction in children and adolescents plays a role in causing hypertension, that is, people with low work and study efficiency and low social interaction are more likely to suffer from hypertension. Li Meng et al.researched that type A behavior is the internal factor of hypertension, and type A behavior means self-centered, demanding others, difficult to establish a harmonious interpersonal relationship with others, less social contact, more likely to produce anxiety, depression and other adverse emotions. This view also confirms the above research findings.

This study shows that residence in rural areas is a risk factor for hypertension, which is consistent with most studies [3]. On the one hand, as living standards improve, rural children may eat more high-calorie, high-salt and high-fat food without the guidance of a balanced diet. On the other hand, this study shows that mothers with high educational background and high academic satisfaction are protective factors for preventing hypertension, while mothers in rural areas with relatively low educational level pay less attention to their children and less investment in educational resources may lead to low academic satisfaction of children. Li's research has also proved that children whose mothers are more educated have higher levels of academic satisfaction [4].

\section{CONCLUSION}

Hypertension is a disease with complex etiology. The occurrence of hypertension in children and adolescents is affected by psychological health status, region, school satisfaction, mother's education background and other psychosocial environmental factors. Therefore,schools and parents should facilitate the mental health education of children and adolescents, impart emotional management and stress coping skills, improve teenagers' social functions, and pay attention to the pressure of academic performance and reduce the mental burden. It is necessary to educate health knowledge and healthy behaviors and habits among children, adolescents and guardians in rural areas, and advocate a reasonable diet so as to reduce the risk of hypertension.

\section{REFERENCES}

[1] Hang Ronghua, Nursing Psychology[M]. Hefei: University of Science and Technology of China Press, 2018.

[2] Yu Dongmei,et al.Blood pressure and hypertension prevalence in children aged 6 to 17 years in China from2010to2012,in:Health Research,2018, pp:1-6.

[3] Wang Shibin,Kou Changgui,Liu Yawen,et al.Rural-urbandifferences in the prevalence of chronic disease in northeastChina, in:Asia Pac J Public Health, 2015, pp:394.

[4] Li Youyou, et al. A survey and analysis of life satisfaction of junior middle school students,in: Journal of Community Medicine, 2017, pp:9-10. 